\title{
Social media use and its impact on adolescent mental health: An umbrella review of the evidence Patti M. Valkenburg ${ }^{1}$, Adrian Meier ${ }^{2}$ and Ine Beyens ${ }^{1}$
}

\begin{abstract}
Literature reviews on how social media use affects adolescent mental health have accumulated at an unprecedented rate of late. Yet, a higher-level integration of the evidence is still lacking. We fill this gap with an up-to-date umbrella review, a review of reviews published between 2019 and mid-2021. Our search yielded 25 reviews: seven meta-analyses, nine systematic, and nine narrative reviews. Results showed that most reviews interpreted the associations between social media use and mental health as 'weak' or 'inconsistent,' whereas a few qualified the same associations as 'substantial' and 'deleterious.' We summarize the gaps identified in the reviews, provide an explanation for their diverging interpretations, and suggest several avenues for future research.
\end{abstract}

\footnotetext{
Addresses

${ }^{1}$ Amsterdam School of Communication Research, University of Amsterdam, Netherlands

${ }^{2}$ School of Business, Economics and Society, FAU ErlangenNuremberg, Germany
}

Corresponding author: Valkenburg, Patti M (p.m.valkenburg@uva.nl)

Current Opinion in Psychology 2022, 44:58-68

Edited by Lydia Krabbendam and Barbara Braams

This review comes from a themed issue on Adolescent Development

For a complete overview see the Issue and the Editorial

Available online 18 August 2021

http://dx.doi.org/10.1016/j.copsyc.2021.08.017

2352-250X/O 2021 The Author(s). Published by Elsevier Ltd. This is an open access article under the CC BY license (http://creativecommons. org/licenses/by/4.0/).

\section{Keywords}

Meta-review, Social networking sites, SNS, Facebook, Instagram, Wellbeing, Depression, Depressive symptoms.

\section{Introduction}

The past years have witnessed a staggering increase in empirical studies into the effects of social media use (SMU) on adolescents' mental health (e.g. [1-3]), defined as the absence of mental illness and the presence of well-being [4]. This rapid increase may be due to at least two reasons. First, SMU occupies an ever-growing

This research was funded by an NWO Spinoza award to the first author. part of adolescents' daily lives, whereas, at the same time, adolescents do not easily accept parental regulation of this use [5]. Second, adolescence is the stage in life in which well-being shows the most fluctuations [6], in which risk-taking is at its peak [7], and in which mental disorders, such as depression, typically emerge [8]. As social media (SM) offer adolescents ample opportunities to engage in risky behaviors, join dubious communities, and interact with strangers outside of parental oversight, it is imaginable that parents, policymakers, and researchers alike want to understand the effects of adolescents' avid SMU on their mental health.

The rapid increase in empirical studies into the effects of SMU on mental health has been paralleled with a comparable increase in literature reviews. Therefore, instead of adding another review of empirical studies, we decided to conduct an umbrella review, also called a meta-review, which is a synthesis of existing literature reviews [9]. Three earlier umbrella reviews have summarized the effects of SMU on mental health [10-12], but two of them did not focus on adolescents, and none included the 19 reviews published in 2020 and 2021. The aims of our umbrella review were to identify and discuss (1) general characteristics of existing reviews, such as the type of review (meta-analytic, systematic, narrative); (2) the conceptualization of SMU and mental health or its constituent outcomes; (3) the interpretation of the effects of SMU on these outcomes (e.g. weak, inconsistent, strong); and (4) the gaps in the evidence base and directions for future research.

\section{Methods}

The first two authors independently conducted literature searches via Google Scholar to find reviews that appeared from 2019 up to July 2021, combining four sets of search terms that correspond with our inclusion criteria (1) 'review,' 'meta-analysis,' or 'synthesis,' (2) 'social media,' 'social networking site,' 'Facebook,' or 'Instagram,' (3) 'well-being,' 'mental health,' or 'psychopathology,' and (4) 'adolescents,' 'youth,' or 'children.' Included studies had to be (1) published reviews that focused on (2) SMU, (3) mental health, and (4) adolescents.

Our operational definition of mental health included indicators of well-being (i.e. happiness, positive affect, 
life satisfaction) and two levels of ill-being, clinical (i.e. depression, anxiety disorder) and non-clinical illbeing (i.e. depressive and anxiety symptoms, distress, negative affect). Because of space restrictions, other indicators and precursors of mental health, such as selfesteem, self-harm, suicidality, loneliness, sleep quality, or externalizing problems, were not considered. We defined SMU as the active (e.g. posting) or passive (e.g. browsing), private (one-to-one) or public (e.g. oneto-many) usage of SM platforms, such as Instagram, Snapchat, Facebook, WeChat, and WhatsApp. Studies focusing on overall 'screen time' were excluded to avoid conceptual conflation of SMU with, for example, television viewing and/or gaming (e.g. [13]).

\section{Results}

Our search yielded 25 reviews, seven meta-analyses, which either included only adolescents [14] or used age as a moderator [15-20]; nine systematic reviews (which reported a systematic search and a synthesis of included studies in tables) [21-29]; and nine narrative reviews [30-38]. Fourteen of these reviews were published in medical/psychiatric journals, eight in psychology journals, and three in social science journals.

\section{Conceptualizations of SMU and well-being}

Tables $1-3$ at the end of this paper list the predictors and outcomes that each of the meta-analytic (Table 1), systematic (Table 2), and narrative reviews (Table 3) mention in their title or abstract. Although all reviews largely relied on the same evidence base, some studies used SMU in the title or abstract, others 'digital media use,' and yet others '(digital) technology use.' Six out of the 25 reviews did not define their predictor. Likewise, 15 reviews failed to define their outcome variables. Some reviews considered well-being as an aspect of mental health [31], whereas others perceived mental health as an aspect of well-being [23]. In addition, several reviews used a broad and sometimes even boundless (operational) definition of mental health, which led to the inclusion of a multitude of outcomes, including marihuana use, identity development, social support, (cyber)bullying, and/or academic performance $[22,23,27,30]$.

\section{Main findings of the reviews}

As Table 1 shows, five meta-analyses yielded associations of general use of social network sites (SNS use) with higher levels of adolescent ill-being that ranged from very small to moderate $(r=.05$ to $r=.17)[14,17-$ 20], and one did not find such an association ( $\mathrm{r}=.02 \mathrm{~ns}$, $15)$. As for well-being, one meta-analysis found that SNS use was weakly associated with higher levels of wellbeing $(\mathrm{r}=+.05)$ [19], whereas another found that it was weakly related to lower levels of well-being $(\mathrm{r}=-.06)$ [17]. However, the latter study aggregated well-being outcomes (e.g. happiness, life satisfaction) with ill-being outcomes (e.g. reversed depression and anxiety scores) in a composite 'well-being' score. When this meta-analysis analyzed happiness, life satisfaction, and depression separately, it found that SNS use was associated with both higher levels of well-being and illbeing [17].

In all, the available meta-analytic evidence suggests that SNS use is weakly associated with higher levels of illbeing $[14,17-20]$ but also with higher levels of wellbeing $[17,19]$, a result that suggests that ill-being is not simply the flip-side of well-being and vice versa, and that both outcomes should be investigated in their own right [11,39]. Finally, all meta-analyses reported considerable variability in the reported associations. For example, in the meta-analysis by Ivie et al. [14], the reported associations of SMU with depressive symptoms ranged from $\mathrm{r}=-.10$ to $\mathrm{r}=+.33$.

While the meta-analyses interpreted the effect sizes predominantly in statistical terms (e.g. small or moderate effect size), the systematic and narrative reviews left more room for diverging interpretations. As Tables 2 and 3 show, most of the conclusions of the 18 systematic and narrative reviews agreed that the effects of SMU are small, and the findings are inconsistent across studies. However, some reviews were less nuanced in their conclusions and used qualifications of the effect sizes such as 'substantial,' 'detrimental,' and 'deleterious' $[25,30,38]$. Some of these reviews also confounded the associations of general time spent with SM with problematic SMU $[21,22,25]$, which is questionable because problematic SMU is a complex phenomenon that entails more than spending a great deal of time with SM. In fact, time spent with SM explains only $6 \%$ of problematic SMU [40]. Problematic SMU is characterized by an enduring preoccupation with SM, an inability to stop using SM, persistent neglect of one's health (e.g. lack of sleep) and important life areas (e.g. family, friends, schoolwork) [40]. For further conclusions of the systematic and narrative reviews, see Tables 2 and 3 .

\section{Identified gaps in the literature and proposed avenues for future research}

As Tables $1-3$ show, 21 out of the 25 reviews agreed that the evidence on which their conclusions are based is primarily cross-sectional so that causal conclusions are not warranted. Other identified gaps involved the lack of attention to mediators to explain the association of SMU with mental health (e.g. [24,32,37]), and the lack of attention to risk and protective factors that may uncover which adolescents are particularly susceptible to the effects of SMU (e.g. $[28,32,37]$ ). Most reviews, therefore, called for longitudinal studies to determine the causal direction of the effects of SMU on mental health (e.g. [14,15], and [22]), as well as for research designed to investigate why and for whom SMU is associated with mental health (e.g. [15], [26], [33]). 
Many reviews observed an over-reliance on self-report measures of SMU and its outcomes (e.g. [21], [33], [37]), which may have introduced various biases. This may necessitate a shift toward more objective measures of SMU, such as log-based measures. Some reviews also noted the typically small and homogenous samples (e.g. [21], [33], [41]) and the lack of attention to the content of SM interactions (e.g. [27], [34], [35]), which is likely a more important predictor than time spent with SM [11]. Another future avenue was to use research methods that distinguish between-person associations from withinperson associations of SMU with mental health [14,27,31]. Finally, more research needs to investigate how SMU can be used to promote mental health among youth [27,34] (see Tables 1-3, for further gaps in the literature).

\section{Discussion}

In this umbrella review, we synthesized the results of 25 recent reviews into the effects of SMU on adolescent mental health. Given that adolescents' SMU is continually changing, it is important to provide regular research updates on this use and its potential effects. In addition to the many important future directions raised in earlier reviews, we discuss three crucial avenues for future research.

\section{Defining SMU, defining mental health}

First, future research needs to consistently define the predictors and outcomes under investigation. Several reviews regularly switched between terms such as digital media use, technology use, and SMU without specifying to which media activities these terms refer. In some studies, emailing and gaming were part of the definitions of SMU, whereas others covered only time spent on SNSs. Such imprecise definitions may greatly hinder our understanding of the effects of SMU on mental health because different types of SMU may lead to different effects on mental health outcomes. For example, time spent on SNS is associated with higher levels of depression [17], whereas emotional connectedness to SNS ('intensity of use') [15] and the number of friends on SNS [16] are unrelated to depression. In the world of SM, everything is rapidly new and rapidly old, and, therefore, it is all the more important to define the specific types of SMU under investigation and to hypothesize how and why these types of SMU could affect mental health outcomes.

Likewise, in several reviews, both mental health and well-being were used as catchall terms that were left undefined, which sometimes led to the discussion of a potpourri of cognitive and affective outcomes that each deserve to be investigated in their own right. Our umbrella review confirmed that similar types of SMU can lead to opposite associations with different mental health outcomes [17]. Both SMU and mental health are highly complex constructs. Although most studies have focused on the associations of SMU with depression or depressive symptoms, all other constituent mental health outcomes, including their risk (e.g. loneliness) and resilience factors (e.g. selfesteem), also deserve our full research attention, provided that they are clearly defined and demarcated from other mental health outcomes.

\section{Capturing the content and quality of SM interactions}

Several reviews have pointed at a need to move away from possibly biased self-report measures toward more objective measures of SMU use, such as log-based measures of time spent with SM. Indeed, self-report measures of time spent with SM correlate only moderately with similar log-based measures $[42,43]$. However, although log-based measures are often seen as the gold standard, they have their own validity threats, such as technical errors and the erroneous tracing of SM apps running in the background when the screen is turned off $[42,43]$. This means that the modest correlations between self-reports and log-based measures could be due to validity issues of self-reports but also of objective measures. More importantly, though, most log-based measures only capture time spent with SM apps, which is just as crude a predictor of mental health as comparable self-report measures. If logging measures only reiterate the 'screen time' approach of most selfreport research, they provide only a limited way forward.

To arrive at a true understanding of the effects of SMU on mental health, future research needs to adopt measures that capture adolescents' responses to specific content or qualities of SM interactions. In experimental settings, this can be realized by using mock SM sites, such as the Truman Platform (https://socialmedialab. cornell.edu/) or the mock SM site developed by Shaw et al. [44]. In non-experimental settings, there are three approaches that can be combined with survey or experience sampling studies: (1) The 'Screenomics' approach developed by Reese et al. [45], which entails end-to-end software that randomly collects screenshots of adolescents' smartphones, and extracts text and images; (2) phone-based mobile sensing [46], which captures sound via the microphone and text entered via the keyboard; and (3) analysis of SM 'data download packages' [47], the archives of SM interactions that each SM user is allowed to download. While each of these methods is promising, they require sophisticated technical skills and specific expertise. Therefore, they can best be achieved in collaborative interdisciplinary projects, which are also better equipped to realize larger samples.

\section{Understanding inconsistent interpretations}

Although the majority of the reviews concluded that the reported associations of SMU with mental health were small to moderate, some others interpreted these associations as serious [30], substantial [48] or detrimental [25]. Such disagreeing interpretations can also be 
Meta-analyses on the associations of social media use (SMU) with adolescent mental health.

\begin{tabular}{|c|c|c|c|c|c|c|c|}
\hline Study & $\begin{array}{c}\text { \# Studies \& } \\
\text { covered years }\end{array}$ & Discipline journal & Outcome $^{a}$ & $\begin{array}{l}\text { Definition } \\
\text { predictor }\end{array}$ & $\begin{array}{l}\text { Definition } \\
\text { outcome }\end{array}$ & $\begin{array}{l}\text { Main results and } \\
\text { interpretations }\end{array}$ & Main gaps in the literature \\
\hline $\begin{array}{l}\text { Cunningham } \\
\text { et al. (2021) }\end{array}$ & $\begin{array}{l}62 \text { studies } \\
(2011-2018)\end{array}$ & Medicine/Psychiatry & $\begin{array}{l}\text { Depressive } \\
\text { symptoms }\end{array}$ & Yes (SNS) & Yes & $\begin{array}{l}r=.02 \mathrm{~ns} \text { (time spent) for } \\
\text { adolescents, based on } \\
\text { moderation analysis } \\
r=.09 \mathrm{~ns} \text { (intensity of use), } \\
\text { not moderated by age } \\
\text { 'Weak,' 'not clinically } \\
\text { meaningful' effects }\end{array}$ & $\begin{array}{l}\text { - Predominantly } \\
\text { cross-sectional evidence } \\
\text { - Over-reliance } \\
\text { on time spent on SM } \\
\text { - Not enough } \\
\text { focus on mediators } \\
\text { or explanations }\end{array}$ \\
\hline Huang (2021) & $\begin{array}{l}123 \text { studies } \\
(2009-2020)\end{array}$ & Psychology & $\begin{array}{l}\text { Well-being and } \\
\text { distress (ill-being) }\end{array}$ & $\begin{array}{l}\text { Yes (online } \\
\text { network size) }\end{array}$ & Yes & $\begin{array}{l}r=.15^{\star} \text { (network size) with } \\
\text { happiness } \\
r=.10^{\star} \text { (network size) with } \\
\text { life satisfaction } \\
r=.01 \mathrm{~ns} \text { (network size) with } \\
\text { depression } \\
\text { No association was } \\
\text { moderated by age } \\
\text { Súbstantially meaningful } \\
\text { relations' }\end{array}$ & $\begin{array}{l}\text { - Little } \\
\text { attention to the } \\
\text { quality of online networks }\end{array}$ \\
\hline $\begin{array}{l}\text { Ivie et al. (2020) } \\
\text { Only adolescents }\end{array}$ & $\begin{array}{l}12 \text { studies } \\
(2012-2019)\end{array}$ & Medicine/Psychiatry & $\begin{array}{l}\text { Depressive } \\
\text { symptoms }\end{array}$ & Yes (SMU) & No & $\begin{array}{l}r=.12^{*} \text { (time spent and } \\
\text { frequency of use) } \\
\text { 'Small effect,' 'high } \\
\text { variability' }\end{array}$ & $\begin{array}{l}\text { - Predominantly } \\
\text { cross-sectional evidence } \\
\text { - Over-reliance } \\
\text { on self-report measures } \\
\text { - Little attention } \\
\text { to within-person effects }\end{array}$ \\
\hline Liu et al. (2019) & $\begin{array}{l}93 \text { studies } \\
(2006-2018)\end{array}$ & Communication & $\begin{array}{l}\text { Psychological } \\
\text { well-being (= } \\
\text { aggregate of life } \\
\text { satisfaction, } \\
\text { happiness, self- } \\
\text { esteem, anxiety, } \\
\text { depression, } \\
\text { stress, and } \\
\text { loneliness) }\end{array}$ & Yes (SNS) & Yes & $\begin{array}{l}r=-.06^{*} \text { (time spent) with } \\
\text { psych. well-being } \\
r=.14^{*} \text { (time spent) with } \\
\text { happiness } \\
r=.09 \mathrm{~ns} \text { (time spent) with } \\
\text { life satisfaction } \\
r=.13^{\star} \text { (time spent) with } \\
\text { depression } \\
\text { No association was } \\
\text { moderated by age } \\
\text { 'No sweeping conclusions' }\end{array}$ & $\begin{array}{l}\text { - Predominantly } \\
\text { cross-sectional evidence } \\
\text { - Little attention } \\
\text { to the quality of SM } \\
\text { interactions }\end{array}$ \\
\hline \multirow[t]{2}{*}{$\begin{array}{l}\text { Vahedi and } \\
\text { Zannella (2021) }\end{array}$} & $\begin{array}{l}55 \text { studies } \\
(2009-2017)\end{array}$ & Psychology & $\begin{array}{l}\text { Depressive } \\
\text { symptoms }\end{array}$ & Yes (SNS) & Yes & $\begin{array}{l}r=.17^{*} \text { (frequency of } \\
\text { checking SNS), not } \\
\text { moderated by age } \\
\text { 'Small positive association' }\end{array}$ & $\begin{array}{l}\text { - Predominantly } \\
\text { cross-sectional evidence } \\
\text { - Most studies } \\
\text { based on undergraduate } \\
\text { student samples }\end{array}$ \\
\hline & & & & & & & (continued on next page) \\
\hline
\end{tabular}




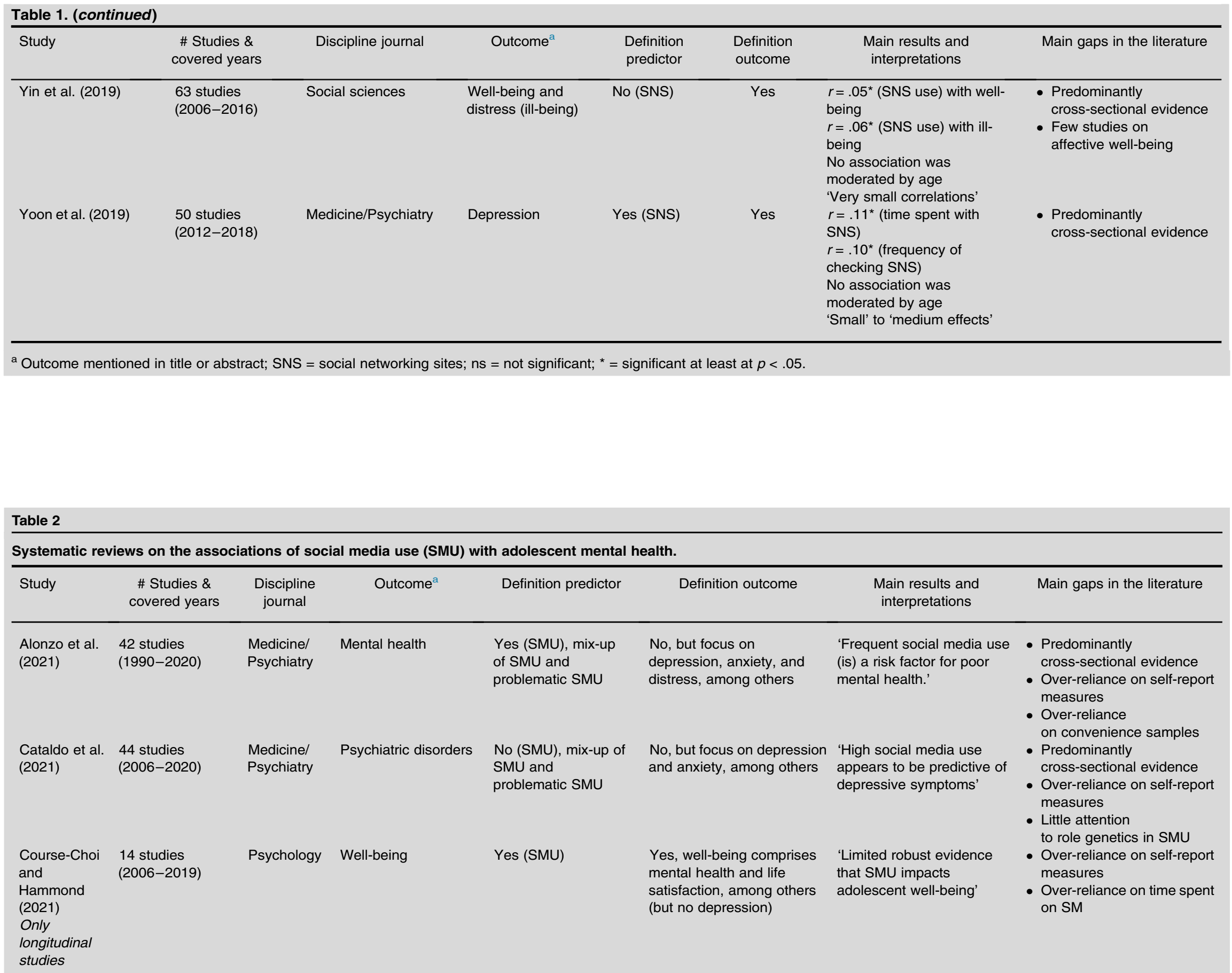




\begin{tabular}{|c|c|c|c|c|c|c|c|}
\hline $\begin{array}{l}\text { Keles et al. } \\
(2020)\end{array}$ & $\begin{array}{l}13 \text { studies } \\
(2011-2018)\end{array}$ & Psychology & $\begin{array}{l}\text { Depression, anxiety, } \\
\text { distress }\end{array}$ & $\begin{array}{l}\text { Yes }(\mathrm{SMU}) \text {, and } \\
\text { problematic SMU }\end{array}$ & $\begin{array}{l}\text { No, but focus on } \\
\text { depression, anxiety, and } \\
\text { distress }\end{array}$ & $\begin{array}{l}\text { Time spent on SM and } \\
\text { problematic use are } \\
\text { 'prominent risk factors' for all } \\
\text { three outcomes. }\end{array}$ & $\begin{array}{l}\text { - Predominantly } \\
\text { cross-sectional evidence } \\
\text { - Over-reliance on self-report } \\
\text { measures } \\
\text { - Little attention } \\
\text { to explanations } \\
\text { - Many studies focus on one } \\
\text { SM }\end{array}$ \\
\hline $\begin{array}{l}\text { Neophytou } \\
\text { et al. (2019) }\end{array}$ & $\begin{array}{l}44 \text { studies } \\
\text { (1999-2019) }\end{array}$ & $\begin{array}{l}\text { Medicine/ } \\
\text { Psychiatry }\end{array}$ & Mental health & $\begin{array}{l}\text { Yes (screen time, } \\
\text { focus on SMU) mix- } \\
\text { up of SMU and } \\
\text { problematic SMU }\end{array}$ & $\begin{array}{l}\text { No, but focus on depression } \\
\text { and anxiety, among many } \\
\text { others }\end{array}$ & $\begin{array}{l}\text { Excessive screen time } \\
(>2-3 \text { h per day), including } \\
\text { SM, 'can have detrimental } \\
\text { effects' on mental health }\end{array}$ & $\begin{array}{l}\text { - Predominantly } \\
\text { cross-sectional evidence } \\
\text { - Over-reliance on self-report } \\
\text { measures }\end{array}$ \\
\hline $\begin{array}{l}\text { Piteo and } \\
\text { Ward (2020) }\end{array}$ & $\begin{array}{l}19 \text { studies } \\
(2005-2019)\end{array}$ & $\begin{array}{l}\text { Medicine/ } \\
\text { Psychiatry }\end{array}$ & $\begin{array}{l}\text { Depressive and } \\
\text { anxiety symptoms }\end{array}$ & $\begin{array}{l}\text { Yes (SNS), includes } \\
\text { problematic SNS use }\end{array}$ & $\begin{array}{l}\text { No, but focus on mental } \\
\text { health, depressive and } \\
\text { anxiety symptoms }\end{array}$ & $\begin{array}{l}\text { 'The effect size tends to be } \\
\text { small and informed by } \\
\text { studies of poor quality.' }\end{array}$ & $\begin{array}{l}\text { - Predominantly } \\
\text { cross-sectional evidence } \\
\text { - Over-reliance on self-report } \\
\text { measures } \\
\text { - Heterogeneity in predictors } \\
\text { and outcomes }\end{array}$ \\
\hline $\begin{array}{l}\text { Schønning } \\
\text { et al. (2020) }\end{array}$ & $\begin{array}{l}79 \text { studies } \\
(2016-2020)\end{array}$ & Psychology & $\begin{array}{l}\text { Mental health and } \\
\text { well-being }\end{array}$ & Yes (SMU) & $\begin{array}{l}\text { No, but focus on broad } \\
\text { range of outcomes, } \\
\text { including depression and } \\
\text { well-being }\end{array}$ & $\begin{array}{l}\text { The relation of SMU and } \\
\text { mental health is complex: } \\
\text { there is 'a culture of fear } \\
\text { around social media, with a } \\
\text { focus on its negative } \\
\text { elements.' }\end{array}$ & $\begin{array}{l}\text { - Predominantly } \\
\text { cross-sectional evidence } \\
\text { - Limited focus on time spent } \\
\text { with SMU } \\
\text { - Stronger focus on negative } \\
\text { than positive effects of } \\
\text { SMU } \\
\text { - Little attention to } \\
\text { within-person effects }\end{array}$ \\
\hline $\begin{array}{l}\text { Vidal et al. } \\
(2020)\end{array}$ & $\begin{array}{l}42 \text { studies } \\
(2011-2019)\end{array}$ & $\begin{array}{l}\text { Medicine/ } \\
\text { Psychiatry }\end{array}$ & Depression & $\begin{array}{l}\text { Yes (SMU), with a } \\
\text { focus on SNS, but } \\
\text { also includes screen } \\
\text { time, problematic } \\
\text { internet use, etc. }\end{array}$ & $\begin{array}{l}\text { No, but focus on } \\
\text { depression, } \\
\text { among others }\end{array}$ & $\begin{array}{l}\text { The majority of studies } \\
\text { 'demonstrate a positive and } \\
\text { bi-directional association } \\
\text { between frequency of SM } \\
\text { use and depression.' }\end{array}$ & $\begin{array}{l}\text { - Predominantly } \\
\text { cross-sectional evidence } \\
\text { - Over-reliance on self-report } \\
\text { measures } \\
\text { - Little attention } \\
\text { to moderators (family } \\
\text { support) } \\
\text { - No clear definitions of SMU } \\
\text { in studies }\end{array}$ \\
\hline $\begin{array}{l}\text { Webster et al. } \\
(2020)\end{array}$ & $\begin{array}{l}23 \text { studies } \\
(1986-2018)\end{array}$ & Sociology & $\begin{array}{l}\text { Subjective well- } \\
\text { being }\end{array}$ & Yes (SMU) & $\begin{array}{l}\text { Yes, focus on mood } \\
\text { and life satisfaction, } \\
\text { among others }\end{array}$ & $\begin{array}{l}\text { Mixed associations across } \\
\text { studies: 'Online social } \\
\text { networks themselves are } \\
\text { not 'bad' for subjective well- } \\
\text { being.' }\end{array}$ & $\begin{array}{l}\text { Little research on } \\
\text { the effects of offline } \\
\text { compared to online } \\
\text { networks on well-being }\end{array}$ \\
\hline
\end{tabular}




\section{Table 3}

Narrative reviews on the associations of social media use (SMU) with adolescent mental health.

\begin{tabular}{|c|c|c|c|c|c|c|}
\hline Study & $\begin{array}{l}\text { Discipline } \\
\text { journal }\end{array}$ & Outcome $^{a}$ & Definition predictor & Definition outcome & $\begin{array}{l}\text { Main results and } \\
\text { interpretations }\end{array}$ & Main gaps in the literature \\
\hline $\begin{array}{l}\text { Abi-Jaoude } \\
\text { et al. (2020) }\end{array}$ & $\begin{array}{l}\text { Medicine/ } \\
\text { Psychiatry }\end{array}$ & $\begin{array}{l}\text { Mental } \\
\text { health }\end{array}$ & $\begin{array}{l}\text { No, but focus on } \\
\text { smartphone and SMU }\end{array}$ & $\begin{array}{l}\text { No, discusses } 30+ \\
\text { outcomes, ranging from } \\
\text { mental distress to } \\
\text { academic performance }\end{array}$ & $\begin{array}{l}\text { SMU leads to increases in } \\
\text { mental distress, and } \\
\text { suicidality among youth; } \\
\text { 'there is a dose-response } \\
\text { relationship.' }\end{array}$ & $\begin{array}{l}\text { - Predominantly } \\
\text { cross-sectional evidence }\end{array}$ \\
\hline $\begin{array}{l}\text { Dienlin and } \\
\text { Johannes } \\
\text { (2020) }\end{array}$ & $\begin{array}{l}\text { Medicine/ } \\
\text { Psychiatry }\end{array}$ & Well-being & $\begin{array}{l}\text { Yes (digital technology use), } \\
\text { includes but is not limited to } \\
\text { SMU }\end{array}$ & $\begin{array}{l}\text { Yes, but discusses a myriad } \\
\text { of other outcomes than } \\
\text { those defined (e.g. ADHD, } \\
\text { academic performance) }\end{array}$ & $\begin{array}{l}\text { Effects are 'likely in the } \\
\text { negative spectrum,', 'but too } \\
\text { small to matter.' }\end{array}$ & $\begin{array}{l}\text { - Over-reliance on } \\
\text { self-report measures } \\
\text { - Little attention } \\
\text { to explanations and } \\
\text { moderators } \\
\text { - Little attention to } \\
\text { within-person effects }\end{array}$ \\
\hline $\begin{array}{l}\text { McLean et al. } \\
\text { (2019) }\end{array}$ & $\begin{array}{l}\text { Medicine/ } \\
\text { Psychiatry }\end{array}$ & Well-being & $\begin{array}{l}\text { Yes (posting and browsing } \\
\text { selfies) }\end{array}$ & $\begin{array}{l}\text { Yes, 'psychological } \\
\text { functioning, such as affect } \\
\text { and self-esteem' }\end{array}$ & $\begin{array}{l}\text { Viewing selfies may } \\
\text { negatively impact well- } \\
\text { being. But research is too } \\
\text { limited to assess the impact } \\
\text { of selfies on well-being. }\end{array}$ & $\begin{array}{l}\text { - Predominantly } \\
\text { cross-sectional evidence } \\
\text { - Little research on children } \\
\text { and preadolescents } \\
\text { - Little research } \\
\text { on buffering and } \\
\text { vulnerability factors }\end{array}$ \\
\hline $\begin{array}{l}\text { Odgers and } \\
\text { Jensen } \\
(2020)[30]\end{array}$ & $\begin{array}{l}\text { Medicine/ } \\
\text { Psychiatry }\end{array}$ & $\begin{array}{l}\text { Mental } \\
\text { health }\end{array}$ & $\begin{array}{l}\text { No, digital technology use, } \\
\text { time online, SNS use are } \\
\text { used interchangeably }\end{array}$ & $\begin{array}{l}\text { No, mental health with a } \\
\text { focus on depression and } \\
\text { anxiety, among many other } \\
\text { outcomes }\end{array}$ & $\begin{array}{l}\text { Small and inconsistent } \\
\text { associations. Even the } \\
\text { associations of the most } \\
\text { rigorous studies 'are unlikely } \\
\text { to be of clinical or practical } \\
\text { significance.' }\end{array}$ & $\begin{array}{l}\text { - Predominantly } \\
\text { cross-sectional evidence } \\
\text { - Over-reliance on } \\
\text { self-report measures } \\
\text { - Small } \\
\text { and nonrepresentative } \\
\text { samples } \\
\text { - Bias towards } \\
\text { high-resource samples }\end{array}$ \\
\hline $\begin{array}{l}\text { Odgers and } \\
\text { Jensen, } 2020 \\
\text { [31] }\end{array}$ & $\begin{array}{l}\text { Medicine/ } \\
\text { Psychiatry }\end{array}$ & $\begin{array}{l}\text { Mental } \\
\text { health }\end{array}$ & $\begin{array}{l}\text { No, digital media use, SMU, } \\
\text { and online engagement are } \\
\text { used interchangeably }\end{array}$ & $\begin{array}{l}\text { No, mental health, well- } \\
\text { being, internalizing } \\
\text { behavior, and depression } \\
\text { are used interchangeably }\end{array}$ & $\begin{array}{l}\text { 'Associations are typically } \\
\text { confounded, with the most } \\
\text { rigorous studies detailing } \\
\text { very small to null } \\
\text { associations.' }\end{array}$ & $\begin{array}{l}\text { - Predominantly } \\
\text { cross-sectional evidence } \\
\text { - Too many studies } \\
\text { on general screen time } \\
\text { - Little attention to potential } \\
\text { positive effects }\end{array}$ \\
\hline
\end{tabular}




\begin{tabular}{|c|c|c|c|c|c|c|}
\hline $\begin{array}{l}\text { Odgers et al. } \\
(2020)\end{array}$ & Psychology & Well-being & $\begin{array}{l}\text { No, digital media use, SMU, } \\
\text { SNS use, and smartphone } \\
\text { use are used } \\
\text { interchangeably }\end{array}$ & $\begin{array}{l}\text { No, social and emotional } \\
\text { well-being and mental } \\
\text { health are used } \\
\text { interchangeably }\end{array}$ & $\begin{array}{l}\text { 'Empirical support for the } \\
\text { story of increasing deficits, } \\
\text { disease, and disconnection } \\
\text { is limited.' }\end{array}$ & $\begin{array}{l}\text { - Predominantly } \\
\text { cross-sectional evidence } \\
\text { - Too much reliance } \\
\text { on screen time measures } \\
\text { - Over-reliance on } \\
\text { self-report measures } \\
\text { - Little attention } \\
\text { to individual differences }\end{array}$ \\
\hline Orben (2020) & $\begin{array}{l}\text { Medicine/ } \\
\text { Psychiatry }\end{array}$ & $\begin{array}{l}\text { Psychological } \\
\text { well-being }\end{array}$ & No, but focus on SMU & $\begin{array}{l}\text { No, outcomes included } \\
\text { depression, social } \\
\text { support, social } \\
\text { connections, life } \\
\text { satisfaction, anxiety, } \\
\text { self-esteem and loneliness }\end{array}$ & $\begin{array}{l}\text { The association is '"negative } \\
\text { but very small.' And 'the } \\
\text { direction is unclear.' }\end{array}$ & $\begin{array}{l}\text { - Predominantly } \\
\text { cross-sectional evidence } \\
\text { - Over-reliance on } \\
\text { self-report measures } \\
\text { - Lack of } \\
\text { transparency (e.g., no } \\
\text { preregistration) } \\
\text { - Little attention } \\
\text { to individual differences }\end{array}$ \\
\hline $\begin{array}{l}\text { Smith et al. } \\
(2021)\end{array}$ & Psychology & Well-being & Yes (SMU) & $\begin{array}{l}\text { No, well-being, emotional } \\
\text { well-being, loneliness, and } \\
\text { belonging are used } \\
\text { interchangeably }\end{array}$ & $\begin{array}{l}\text { The relationships 'are } \\
\text { multifaceted and complex.' }\end{array}$ & $\begin{array}{l}\text { - Predominantly } \\
\text { cross-sectional evidence } \\
\text { - Little attention } \\
\text { to explanations } \\
\text { - Little attention to cultura } \\
\text { differences }\end{array}$ \\
\hline $\begin{array}{l}\text { Twenge } \\
\text { (2019) }\end{array}$ & Psychology & $\begin{array}{l}\text { Depression } \\
\text { symptoms }\end{array}$ & $\begin{array}{l}\text { No, technology use, digital } \\
\text { media use and SMU are } \\
\text { used interchangeably }\end{array}$ & $\begin{array}{l}\text { No, depressive symptoms, } \\
\text { mental health, } \\
\text { psychological well-being } \\
\text { are used interchangeably }\end{array}$ & $\begin{array}{l}\text { Associations are } \\
\text { 'considerable' and } \\
\text { 'substantial.' }\end{array}$ & $\begin{array}{l}\text { - Predominantly } \\
\text { cross-sectional evidence } \\
\text { - Only research at } \\
\text { the individual level and } \\
\text { not at the collective level }\end{array}$ \\
\hline
\end{tabular}

${ }^{\text {a }}$ Outcome mentioned in title or abstract. 
witnessed in three recent publications on SMU and mental health by Twenge et al. [49], Orben and Przybylski [3], and Kreski et al. [50], all relying on the same UK-based data set. Such divides in interpretations of the same modest effect sizes are certainly not new in the media effects field. For example, as of the 1980s, there has been a fierce debate among scholars about the effects of game violence on aggression (e.g. see the dispute in Psychological Bulletin about whether this effect is trivial or meaningful [51,52]). Oftentimes, the involved scholars do not disagree that much about the size of the reported effects but just on how to interpret them.

What has often been ignored in such debates is that the effect sizes are just what they are: statistics observed at the aggregate level. Such statistics are typically derived from heterogeneous samples of adolescents who may differ greatly in their susceptibilities to the effects of environmental influences in general [53] and media influences in particular [54]. After all, each adolescent is subject to unique dispositional, social-context, and situational factors that guide their SMU and moderate its effects [55]. Such person-specific antecedents and effects of SMU cannot be captured by the aggregatelevel statistics that have been reported in the majority of empirical studies and reviews, including the current one.

If we accept the propositions of media-specific susceptibility theories [54], it is plausible to assume that both optimistic and pessimistic conclusions about the effects of SMU are valid - they just refer to different adolescents. In fact, recent studies that have adopted an idiographic (i.e. $N=1$ or person-specific) media effects paradigm [56] have found that a small group of adolescents experienced negative effects of SMU on wellbeing (around 10-15\%) and another small group experienced positive effects (also around 10\%-15\%). Reassuringly though, most adolescents experienced no or negligible effects [57].

A person-specific approach to media effects requires a large number of respondents and a large number of withinperson observations per respondent. Indeed, statistical power is expensive. However, due to rapidly advancing technological (e.g. phone-based experience sampling methods) and methodological developments (e.g. $N=1$ time series analyses), such approaches are increasingly within everyone's reach, especially when researchers pool resources in interdisciplinary teams. A person-specific media effects paradigm may not only help academics resolve controversies between optimistic and pessimistic interpretations of aggregate-level effect sizes, but it may also help us understand when, why, and for whom SMU can lead to positive or negative effects on mental health. And above all, it may help us facilitate personalized prevention and intervention strategies to help adolescents maintain or improve their mental health.

\section{Credit author statement}

Patti M. Valkenburg: Conceptualization, Literature search; Creating tables; Writing paper; Adrian Meier: Literature search; Commenting on draft versions of paper; Checking tables; Ine Beyens: Commenting on draft versions of paper; Checking tables.

\section{Conflict of interest statement}

None of the authors declared a conflict of interest.

\section{References}

Papers of particular interest, published within the period of review, have been highlighted as:

* of special interest

** of outstanding interest

1. Beyens I, Pouwels JL, van Driel II, Keijsers L, Valkenburg PM: ** The effect of social media on well-being differs from adolescent to adolescent. Sci Rep 2020, 10:10763.

This is the first study showing that the effect of social media use differs from adolescent to adolescent. It is also among the first to disconfirm the hypothesis that passive social media use (i.e., browsing) is negatively associated with well-being. It found, for example, that $46 \%$ of adolescents felt happier after browsing, whereas only $10 \%$ of adolescents felt less happy after browsing. The remaining $44 \%$ did not feel more or less happy after browsing.

2. Jensen M, George MJ, Russell MR, Odgers CL: Young adolescents' digital technology use and mental health symptoms: little evidence of longitudinal or daily linkages. Clin Psychol Sci 2019, 7:1416-1433.

This study demonstrates that it is important to distinguish within-person associations from between-person associations among adolescents. Investigating within-person associations is important when investigating the effects of (social) media use, because media effects are withinperson changes due to (social) media use, and such changes can best be investigated by means of within-person methods of analysis.

3. Orben A, Przybylski AK: The association between adolescent well-being and digital technology use. Nature Human Behav 2019, 3:173-182.

4. Keyes CLM, Lopez SJ: Toward a science of mental health. In Oxford handbook of positive psychology. Edited by Snyder CR, Lopez SJ, New York: Oxford University Press; 2009:45-59.

5. Valkenburg PM, Piotrowski JT: Plugged in: how media attract and affect youth. New Haven, NJ: Yale University Press; 2017.

6. Maciejewski DF, Keijsers L, van Lier PAC, Branje SJT, Meeus WHJ, Koot HM: Most fare well-But some do not: distinct profiles of mood variability development and their association with adjustment during adolescence. Dev Psychol 2019, 55:434-448.

7. Steinberg L: Risk taking in adolescence: new perspectives from brain and behavioral science. Curr Dir Psychol Sci 2007. $16: 55-59$

8. Paus T, Keshavan M, Giedd JN: Why do many psychiatric disorders emerge during adolescence? Nat Rev Neurosci 2008, 9:947-957

9. Aromataris E, Fernandez R, Godfrey CM, Holly C, Khalil H, Tungpunkom P: Summarizing systematic reviews: methodological development, conduct and reporting of an umbrella review approach. JBI Evid Implement 2015, 13:132-140.

10. Appel M, Marker C, Gnambs T: Are social media ruining our lives? A review of meta-analytic evidence. Rev Gen Psychol 2020, 24:60-74.

11. Meier A, Reinecke L: Computer-mediated communication, social media, and mental health: a conceptual and empirical meta-review. Commun Res 2020.

This meta-review proposes two comprehensive frameworks that help organize the vast and conceptually diverse literature on social media and mental health. The Extended Two-Continua Model of Mental 
Health integrates clinical and positive psychology perspectives on mental well- and ill-being. The Taxonomy of Computer-Mediated Communication integrates key approaches to the study of social media effects by distinguishing six main levels of analysis.

12. Arias-de la Torre J, Puigdomenech E, García X, Valderas JM, Eiroa-Orosa FJ, Fernández-Villa T, Molina AJ, Martín V, SerranoBlanco A, Alonso J, Espallargues M: Relationship between depression and the use of mobile technologies and social media among adolescents: umbrella review. J Med Internet Res 2020, 22, e16388.

13. Stiglic N, Viner RM: Effects of screentime on the health and well-being of children and adolescents: a systematic review of reviews. BMJ Open 2019, 9, e023191.

14. Ivie EJ, Pettitt A, Moses LJ, Allen NB: A meta-analysis of the * association between adolescent social media use and depressive symptoms. J Affect Disord 2020, 275:165-174.

This is, to our knowledge, the only meta-analysis that focused on adolescents. Most of the remaining meta-analyses included both adults and adolescents and did not find moderating effects of age. This lack of moderation effects could, however, be due to the relatively low number of studies on adolescents in these meta-analyses, which may have caused power problems. Moreover, most studies on adults have focussed on young adults (e.g., students), which has introduced a rather restricted age range into this literature. As Table 1 shows, Ivie et al. reported a modest effect size of social media use on depressive symptoms

15. Cunningham S, Hudson CC, Harkness K: Social media and depression symptoms: a meta-analysis. Res Child Adolesc Psychopathol 2021, 49:241-253.

16. Huang C: Correlations of online social network size with wellbeing and distress: a meta-analysis. Cyberpsychology 2021, 15,3 .

17. Liu D, Baumeister RF, Yang C-c, Hu B: Digital communication media use and psychological well-being: a meta-analysis. J Computer-Mediated Commun 2019, 24:259-274.

18. Vahedi Z, Zannella L: The association between self-reported depressive symptoms and the use of social networking sites (sns): a meta-analysis. Curr Psychol: J Div Perspect Div Psychol Issu 2021, 40:2174-2189.

19. Yin X-Q, De Vries DA, Gentile DA, Wang J-L: Cultural background and measurement of usage moderate the association between social networking sites (SNSs) usage and mental health: a meta-analysis. Soc Sci Comput Rev 2019, 37:631-648.

20. Yoon S, Kleinman M, Mertz J, Brannick M: Is social network site usage related to depression? A meta-analysis of Facebook-depression relations. J Affect Disord 2019, 248: 65-72.

21. Alonzo R, Hussain J, Stranges S, Anderson KK: Interplay between social media use, sleep quality, and mental health in youth: a systematic review. Sleep Med Rev 2021, 56:101414.

22. Cataldo I, Lepri B, Neoh MJY, Esposito G: Social media usage and development of psychiatric disorders in childhood and adolescence: a review. Front Psychiatr 2021, 11.

23. Course-Choi J, Hammond L: Social media use and adolescent well-being: a narrative review of longitudinal studies. Cyberpsychol, Behav Soc Netw 2021, 24:223-236.

24. Keles B, McCrae N, Grealish A: A systematic review: the influence of social media on depression, anxiety and psychological distress in adolescents. Int J Adolesc Youth 2020, 25:79-93.

25. Neophytou E, Manwell LA, Eikelboom R: Effects of excessive screen time on neurodevelopment, learning, memory, menta health, and neurodegeneration: a scoping review. Int J Ment Health Addiction 2019, 19:724-744.

26. Piteo EM, Ward K: Social networking sites and associations with depressive and anxiety symptoms in children and adolescents: a systematic review. Child Adolesc Ment Health 2020 25:201-216.

27. Schønning V, Hjetland GJ, Aarø LE, Skogen JC: Social media use and mental health and well-being among adolescents: a scoping review. Front Psychol 2020, 11:1949.
28. Vidal C, Lhaksampa T, Miller L, Platt R: Social media use and depression in adolescents: a scoping review. Int Rev Psychiatr 2020, 32:235-253.

29. Webster D, Dunne L, Hunter R: Association between social networks and subjective well-being in adolescents: a systematic review. Youth Soc 2020, 53:175-210.

30. Abi-Jaoude E, Naylor KT, Pignatiello A: Smartphones, social media use and youth mental health. Can Med Assoc J 2020, 192, E136.

31. Dienlin $\mathrm{T}$, Johannes $\mathrm{N}$ : The impact of digital technology use on adolescent well-being. Dialogues Clin Neurosci 2020, 22: 135-142.

32. McLean SA, Jarman HK, Rodgers RF: How do "selfies" impact adolescents' well-being and body confidence? A narrative review. Psychol Res Behav Manag 2019, 12:513-521.

33. Odgers $C L$, Jensen MR: Annual research review: adolescent * mental health in the digital age: facts, fears, and future directions. JCPP (J Child Psychol Psychiatry) 2020, 61: $336-348$.

This is an extensive narrative review on adolescents' social media use and mental health. Its conclusions are nuanced and balanced, and it provides many important avenues for future research (see also Table 3).

34. Odgers CL, Jensen MR: Adolescent development and growing divides in the digital age. Dialogues Clin Neurosci 2020, 22: 143-149.

35. Odgers CL, Schueller SM, Ito M: Screen time, social media use, and adolescent development. Ann Rev Develop Psychol 2020, 2: 485-502.

36. Smith D, Leonis T, Anandavalli S: Belonging and loneliness in cyberspace: impacts of social media on adolescents' wellbeing. Aust J Psychol 2021, 73:12-23.

37. Orben A: Teenagers, screens and social media: a narrative review of reviews and key studies. Soc Psychiatr Psychiatr Epidemiol 2020, 55:407-414.

38. Twenge JM: More time on technology, less happiness? Associations between digital-media use and psychological wellbeing. Curr Dir Psychol Sci 2019, 28:372-379.

39. Ryff CD, Dienberg Love G, Urry HL, Muller D, Rosenkranz MA, Friedman EM, Davidson RJ, Singer B: Psychological well-being and ill-Being: do they have distinct or mirrored biological correlates? Psychother Psychosom 2006, 75:85-95.

40. van den Eijnden RJJM, Lemmens JS, Valkenburg PM: The social media disorder scale. Comput Hum Behav 2016, 61: $478-487$.

41. Vahedi Z, Zannella L: The association between self-reported depressive symptoms and the use of social networking sites (sns): a meta-analysis. Curr Psychol: J Div Perspect Div Psychol Issu 2021, 40:2174-2189.

42. Parry DA, Davidson BI, Sewall CJR, Fisher JT, Mieczkowski H, Quintana DS: A systematic review and meta-analysis of discrepancies between logged and self-reported digital media use. Nature Human Behav 2021.

This is a recent meta-analysis that compared self-report measures and objective measures of time spent with social media. It gives a balanced view on the pros and cons of objective measures.

43. Verbeij T, Pouwels JL, Beyens I, Valkenburg PM: The accuracy and validity of self-reported social media use measures among adolescents. Comput Human Behav Rep 2021, 3:100090.

44. Shaw DJ, Pennington CR, Ngombe N, Kessler K, Kaye LK: It's not what you do, it's the way that you do it: an experimental task delineates among styles of behaviour on social networking sites and psychosocial measures. PsyArXiv; 2021.

45. Reeves B, Ram N, Robinson TN, Cummings JJ, Giles CL, Pan J, Chiatti A, Cho M, Roehrick K, Yang X, Gagneja A, Brinberg M, Muise D, Lu Y, Luo M, Fitzgerald A, Yeykelis L: Screenomics: a framework to capture and analyze personal life experiences and the ways that technology shapes them. Hum Comput Interact 2021, 36:150-201. 
46. Harari GM, Müller SR, Gosling SD. In Naturalistic assessment of situations using mobile sensing methods. Edited by Widiger TH, Oxford University Press; 2020:299-311.

This is a good review chapter on mobile sensing methods. It compares different mobile sensing methods, and discusses the pros and cons of mobile sensing to capture the content and quality of social media interactions.

47. Boeschoten L, Ausloos J, Moeller J, Araujo T, Oberski DL: Digital trace data collection through data donation. arXiv; 2020. preprint arXiv:2011.09851.

48. Twenge JM: Why increases in adolescent depression may be linked to the technological environment. Curr Opin Psychol 2020, 32:89-94.

49. Twenge JM, Joiner TE, Rogers ML, Martin GN: Increases in depressive symptoms, suicide-related outcomes, and suicide rates among U.S. Adolescents after 2010 and links to increased new media screen time. Clin Psychol Sci 2018, 6:3-17.

50. Kreski N, Platt J, Rutherford C, Olfson M, Odgers C Schulenberg J, Keyes KM: Social media use and depressive symptoms among United States adolescents. J Adolesc Health 2021, 68:572-579.

51. Bushman BJ, Rothstein HR, Anderson CA: Much ado about something: violent video game effects and a school of red herring: reply to Ferguson and Kilburn (2010). Psychol Bull 2010, 136:182-187.

52. Ferguson CJ, Kilburn J: Much ado about nothing: the misestimation and overinterpretation of violent video game effects in eastern and western nations: comment on Anderson et al. (2010). Psychol Bull 2010, 136:174-178.

53. Belsky J, Pluess M: Beyond diathesis stress: differential susceptibility to environmental influences. Psychol Bull 2009 135:885-908.

54. Valkenburg PM, Peter J: The differential susceptibility to media effects model. J Commun 2013, 63:221-243.

55. Valkenburg PM, Peter J, Walther JB: Media effects: theory and research. Annu Rev Psychol 2016, 67:315-338.

56. Valkenburg PM, Beyens I, Pouwels JL, van Driel II, Keijsers L: Social media and adolescents' self-esteem: heading for a person-specific media effects paradigm. J Commun 2021, $71: 56-78$

57. Valkenburg PM, Beyens I, Pouwels JL, van Driel II, Keijsers L: Social media browsing and adolescent well-being: challenging the "passive social media use hypothesis". J Computer-Mediated Commun 2021.

This is the first study that used a person-specific approach to explain why social media browsing leads to increases in well-being among some adolescents and decreases in well-being among others. It shows, for example, that adolescents more often experienced a negative effect of browsing on well-being when they felt envy during browsing, and that they more often experienced a positive effect when they enjoyed their social media experience. 